\title{
A New Era of Systemic Therapy for Hepatocellular Carcinoma with Regorafenib and Lenvatinib
}

Prof. M. Kudo

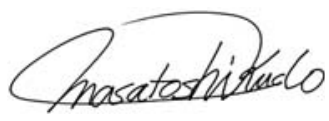

Editor Liver Cancer

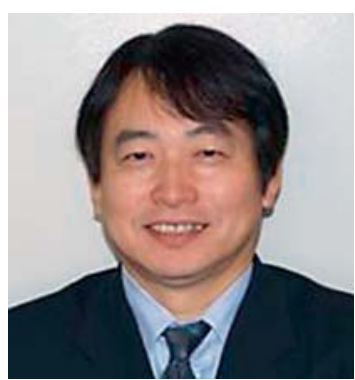

\section{Introduction}

The SHARP study in 2007 [1] and the Asia Pacific study in 2008 [2] led to the worldwide approval of sorafenib as the standard therapy for unresectable hepatocellular carcinoma (HCC). However, clinical trials investigating sunitinib [3], brivanib [4], and linifanib [5] as first-line treatments, with sorafenib as a control arm, failed to meet their primary endpoint of improving overall survival (OS). In addition, global phase III studies investigating secondline therapy with brivanib [6], everolimus [7], and ramucirumab [8], and a regional trial investigating S-1 (tegafur/gimeracil/oteracil) in Japan, were unsuccessful (Table 1). Thus, sorafenib was the only systemic therapeutic agent available for HCC, and for some time no options were available for patients with progressive disease or those intolerant to sorafenib. However, between June 2016 and January 2017, promising results of global phase III studies were reported. The results of a trial of regorafenib were reported at a conference [9] and in a journal [10], and results with lenvatinib were reported in a press release [11], indicating the arrival of a new era of liver cancer therapy.

\section{Sorafenib-Regorafenib Sequential Therapy}

Sorafenib is the standard treatment for advanced-stage HCC (Barcelona Clinic Liver Cancer [BCLC] stage C) and the only therapeutic agent for advanced-stage HCC with vascular invasion and/or extrahepatic spread. However, in addition to patients with advanced-stage 
Kudo et al.: A New Era of Systemic Therapy for Hepatocellular Carcinoma with Regorafenib and Lenvatinib

Table 1. Phase III clinical trials for HCC

\begin{tabular}{|c|c|c|c|c|c|}
\hline $\begin{array}{l}\text { Target } \\
\text { population }\end{array}$ & & Design & Trial name & Presentation & Publication \\
\hline \multicolumn{6}{|l|}{ Early } \\
\hline Adjuvant & 1 & Peretinoin vs. placebo ${ }^{\mathrm{a}}$ & NIK-333 & ASCO 2010 & JG 2014 \\
\hline \multirow{2}{*}{$\begin{array}{l}\text { (prevention of } \\
\text { recurrence) }\end{array}$} & 2 & Sorafenib vs. placebo ${ }^{\mathrm{a}}$ & STORM & ASCO 2014 & Lancet-0 2015 \\
\hline & 3 & Peretinoin vs. placebo & NIK-333/K-333 & Ongoing & \\
\hline \multicolumn{6}{|l|}{ Intermediate } \\
\hline Improvement & 1 & TACE +/- sorafenib ${ }^{\mathrm{a}}$ & Post-TACE & ASCO-GI 2010 & EJC 2011 \\
\hline \multirow{2}{*}{ of TACE } & 2 & TACE +/- brivanib ${ }^{\mathrm{a}}$ & BRISK-TA & ILCA 2013 & Hepatol 2014 \\
\hline & 3 & TACE +/- orantinib ${ }^{\mathrm{a}}$ & ORIENTAL & EASL 2015 & \\
\hline \multicolumn{6}{|l|}{ Advanced } \\
\hline \multirow[t]{6}{*}{ First line } & 1 & Sorafenib vs. sunitinib ${ }^{a}$ & SUN1170 & ASCO 2011 & JCO 2013 \\
\hline & 2 & Sorafenib vs. brivanib ${ }^{a}$ & BRISK-FL & AASLD 2012 & JCO 2013 \\
\hline & 3 & Sorafenib vs. linifanib ${ }^{a}$ & LiGHT & ASCO-GI 2013 & JCO 2015 \\
\hline & 4 & Sorafenib +/- HAIC ${ }^{\mathrm{a}}$ & SILIUS & EASL 2016 & \\
\hline & 5 & Sorafenib vs. lenvatinib & REFLECT & TBD & \\
\hline & 6 & Sorafenib vs. nivolumab & CheckMate 459 & Ongoing & \\
\hline \multirow[t]{8}{*}{ Second line } & 1 & Brivanib vs. placebo $^{a}$ & BRISK-PS & EASL 2012 & JCO 2013 \\
\hline & 2 & Everolimus vs. placebo ${ }^{a}$ & EVOLVE-1 & ASCO-GI 2014 & JAMA 2014 \\
\hline & 3 & Ramucirumab vs. placebo ${ }^{a}$ & REACH & ESMO 2014 & Lancet-0 2015 \\
\hline & 4 & S-1 vs. placebo ${ }^{\mathrm{a}}$ & S-CUBE & ASCO-GI 2015 & \\
\hline & 5 & Regorafenib vs. placebo ${ }^{b}$ & RESORCE & WCGI 2016 & Lancet 2017 \\
\hline & 6 & Tivantinib vs. placebo & JET-HCC & Ongoing & \\
\hline & 7 & Ramucirumab vs. placebo & REACH-2 & Ongoing & \\
\hline & 8 & Pembrolizumab vs. placebo & KEYNOTE-240 & Ongoing & \\
\hline
\end{tabular}

HCC, hepatocellular carcinoma; TACE, transarterial chemoembolization; HAIC, hepatic arterial infusion chemotherapy. ${ }^{\mathrm{a}}$ Trial halted or with negative results. ${ }^{\mathrm{b}}$ Trial with positive results.

HCC (BCLC-C), patients with intermediate-stage HCC (BCLC-B) were included in the SHARP study and the Asia Pacific study; therefore, sorafenib is a widely approved agent for systemic therapy not only for advanced-stage HCC but also for unresectable HCC (including intermediate-stage HCC). In fact, sorafenib is often administered to patients with intermediate-stage HCC (BCLC-B) in clinical practice and to $16.4-19.6 \%$ of patients in clinical trials worldwide [3-5].

The population of patients with intermediate-stage HCC is extremely heterogeneous, comprising 3 main subpopulations: (1) patients with a good prognosis who can be treated with curative therapy, (2) those for whom transarterial chemoembolization (TACE) is indicated, and (3) those for whom TACE is not recommended. This subclassification of intermediate-stage HCC recently became a well-discussed issue, and the Kinki criteria are used to subclassify patients with intermediate-stage HCC $[12,13]$. The OS of patients with B1, B2, and B3 HCC according to the Kinki criteria corresponds to that of patients with HCC of BCLC stages A, B, and C. In addition, patients with BCLC substage B2 HCC, especially those with multiple HCC tumors in both lobes, are not good candidates for TACE. For patients with intermediatestage HCC (BCLC-B) who do not respond to TACE, an early switch to sorafenib therapy (rather than repeat ineffective TACE) results in a better survival benefit; therefore, early identification of patients that are refractory to TACE is crucial [14-17]. These data underscore the importance of establishing globally recognized, common criteria for identifying TACE failure/ refractory patients in order to improve the survival of HCC patients [18]. 


\section{Liver Cancer}

\begin{tabular}{l|l}
\hline \multicolumn{2}{l}{ Liver Cancer 2017;6:177-184 } \\
\hline DOI: 10.1159/000462153 & $\begin{array}{l}\text { C 2017 S. Karger AG, Basel } \\
\text { www.karger.com/lic }\end{array}$ \\
\hline
\end{tabular}

Kudo et al.: A New Era of Systemic Therapy for Hepatocellular Carcinoma with Regorafenib and Lenvatinib

Table 2. Results of the RESORCE trial

\begin{tabular}{lccc}
\hline & Regorafenib & Placebo & HR (95\% CI); $p$ value \\
\hline Subjects, $n$ & 379 & 194 & \\
BCLC stage C, \% & 86 & 89 & \\
Treatment duration, months & $3.6(1.6-7.6)$ & $1.9(1.4-3.9)$ & \\
OS, months & $10.6(9.1-12.1)$ & $7.8(6.3-8.8)$ & $0.63(0.50-0.79) ; p<0.0001$ \\
PFS (mRECIST), months & $3.1(2.8-4.2)$ & $1.5(1.4-1.6)$ & $0.46(0.37-0.56) ; p<0.0001$ \\
TTP (mRECIST), months & $3.2(2.9-4.2)$ & $1.5(1.4-1.6)$ & $0.44(0.36-0.55) ; p<0.0001$ \\
DCR, \% & 65 & 36 & $p<0.0001$ \\
ORR, \% & 11 & 4 & $p=0.0047$ \\
Adverse events (grade $\geq 3), \%$ & 79.7 & 58.5 & \\
\hline
\end{tabular}

Values are presented as median (range) unless specified otherwise. BCLC, Barcelona Clinic Liver Cancer; OS, overall survival; PFS, progression-free survival; TTP, time to progression; DCR, disease control rate; ORR, objective response rate.

The results of the RESORCE study were published recently [10]. The success of the study is attributed to the following 3 points: (1) patients for whom sorafenib therapy was terminated because of adverse events were excluded from the trial, and second-line therapy with regorafenib was provided to HCC patients who had significant disease progression during sorafenib therapy; (2) vascular invasion and extrahepatic spread were considered as separate stratification factors to avoid imbalance in the 2 arms (test agent vs. placebo); and (3) patients showing tolerance to sorafenib (patients who tolerated $400 \mathrm{mg}$ sorafenib daily for a minimum of 20 days within the last 28 days before radiological progression) were enrolled. This trial design prevented the risk of dropouts due to adverse events associated with regorafenib (since the toxicity of regorafenib is similar to that of sorafenib) and minimized the effect of post-trial treatment after disease progression [18]. According to the results of the RESORCE trial, the median OS (mOS) of patients treated with regorafenib was 10.6 months (7.8 for placebo; HR 0.63; $p<0.0001$ ) (Table 2). Subgroup analysis revealed that the HR of OS in patients with a Child-Pugh score of 5 was significantly better than that in patients with a Child-Pugh score of 6 ( 0.60 [0.40-0.79] vs. 0.80 [0.57-1.13]). This also supports the following approach to improving prognosis in the future: an early switch to sorafenib when patients become refractory to TACE and still have good liver function, such as with a Child-Pugh score of 5 , with a subsequent switch to regorafenib as soon as possible after patients have shown progressive disease on sorafenib.

An exploratory analysis of the RESORCE trial showed a favorable OS of 26 months from the start of sorafenib therapy among patients who received sorafenib-regorafenib sequential therapy (19.2 months in the placebo group) [19]. These are extremely important data, because the 26-month prognosis is comparable with the conventional figure after TACE for patients with intermediate-stage HCC [20]. To date, 3 prospective phase III trials evaluating combination therapy with sorafenib and TACE have been performed (Table 1). The results of 2 of these trials have been published: the Japan-Korea TACE study $(n=450)$ [21] and the brivanibTACE (BRISK-TA) study, which evaluated adjuvant brivanib therapy in combination with TACE $(n=502)$ [20]. Because the mOS was not reached in the placebo arm in the post-TACE study, the BRISK-TA study is currently the only prospective phase III study that investigated the OS with TACE and provided a valid $\mathrm{mOS}$ for the placebo arm. Since this was a randomized controlled trial (RCT) of TACE (with the largest number of patients included in any trial worldwide) and the placebo arm was free from selection bias, it provides standard outcomes for TACE worldwide. Additionally, most (82\%) of the patients in the BRISK-TA study had 
Kudo et al.: A New Era of Systemic Therapy for Hepatocellular Carcinoma with Regorafenib and Lenvatinib

Table 3. Comparison of mOS between TACE and sorafenib-regorafenib sequential therapy

\begin{tabular}{lll}
\hline & $\begin{array}{l}\text { TACE (placebo arm of } \\
\text { brivanib-TACE trial) [20] }\end{array}$ & $\begin{array}{l}\text { Sorafenib-regorafenib sequential } \\
\text { therapy (RESORCE trial) }[10,19]\end{array}$ \\
\hline Subjects, $n$ & 253 & 379 \\
Child-Pugh class, $n$ (\%) & & \\
A & $231(91)$ & $373(98)$ \\
B & $20(8)$ & $5(1)$ \\
C & $2(1)$ & $0(0)$ \\
BCLC stage, $n$ (\%) & $57(23)$ & $1(0<1)$ \\
A & $150(59)$ & $53(14)$ \\
B & $44(17)$ & $325(86)$ \\
C & $2(1)$ & $0(0)$ \\
D & 26.1 & 26 \\
mOS, months & &
\end{tabular}

mOS, median overall survival; TACE, transarterial chemoembolization; BCLC, Barcelona Clinic Liver Cancer.

early- or intermediate-stage HCC (BCLC-B, 59\%; BCLC-A, 23\%) and only 17\% had advancedstage HCC. Conversely, $86 \%$ of the patients in the RESORCE trial had advanced-stage HCC (BCLC-C), even though the detailed status of BCLC stage at the start of sorafenib therapy is not disclosed. A simple comparison of OS between the 2 cohorts in different RCTs shows similar outcomes: 26.1 months for the control arm of BRISK-TA versus 26 months for sorafenib-regorafenib sequential therapy (Table 3). Although comparing one arm from an RCT with an arm from exploratory analysis of a different RCT may not be appropriate, both were well-designed prospective randomized clinical trials that minimized the effect of selection bias. Considering that the patients in the sorafenib-regorafenib sequential therapy trial overwhelmingly had advanced-stage HCC, the OS in the study, which is comparable to that in BRISK-TA, is extremely impressive. There is no doubt that the group of subjects with advanced-stage HCC were superselected; nevertheless, the effect of sorafenib-regorafenib sequential therapy in patients with advanced-stage HCC is comparable to that of TACE in those for whom TACE is indicated.

Since the prognosis after sorafenib-regorafenib sequential therapy is promising, the appropriate time to start sorafenib therapy may need to be reconsidered. Conventionally, patients are switched from TACE to systemic therapy when they become refractory to TACE; however, a more proactive approach, such as the earlier introduction of systemic therapy to subgroups of patients who are likely to become refractory to TACE when their liver function is still preserved (Child-Pugh A), may be beneficial (Fig. 1).

Another unaddressed question is the proportion of patients who can be treated by sorafenib-regorafenib sequential therapy. In other words, even after the approval of regorafenib, further studies are needed to obtain real-world data on the percentage of patients undergoing sorafenib therapy who can complete the therapy and also complete regorafenib therapy after failure of sorafenib treatment and disease progression. However, the optimal time point to determine TACE refractoriness should not be missed, since the prognosis after sorafenib-regorafenib sequential therapy is good (26 months from the initiation of sorafenib therapy). Early identification of subgroups likely to become refractory to TACE is also important (Fig. 2). The selection of patients who are likely to respond to systemic therapy is a valid and important line of inquiry regarding therapy with other agents, including lenvatinib (discussed below) and sorafenib. 


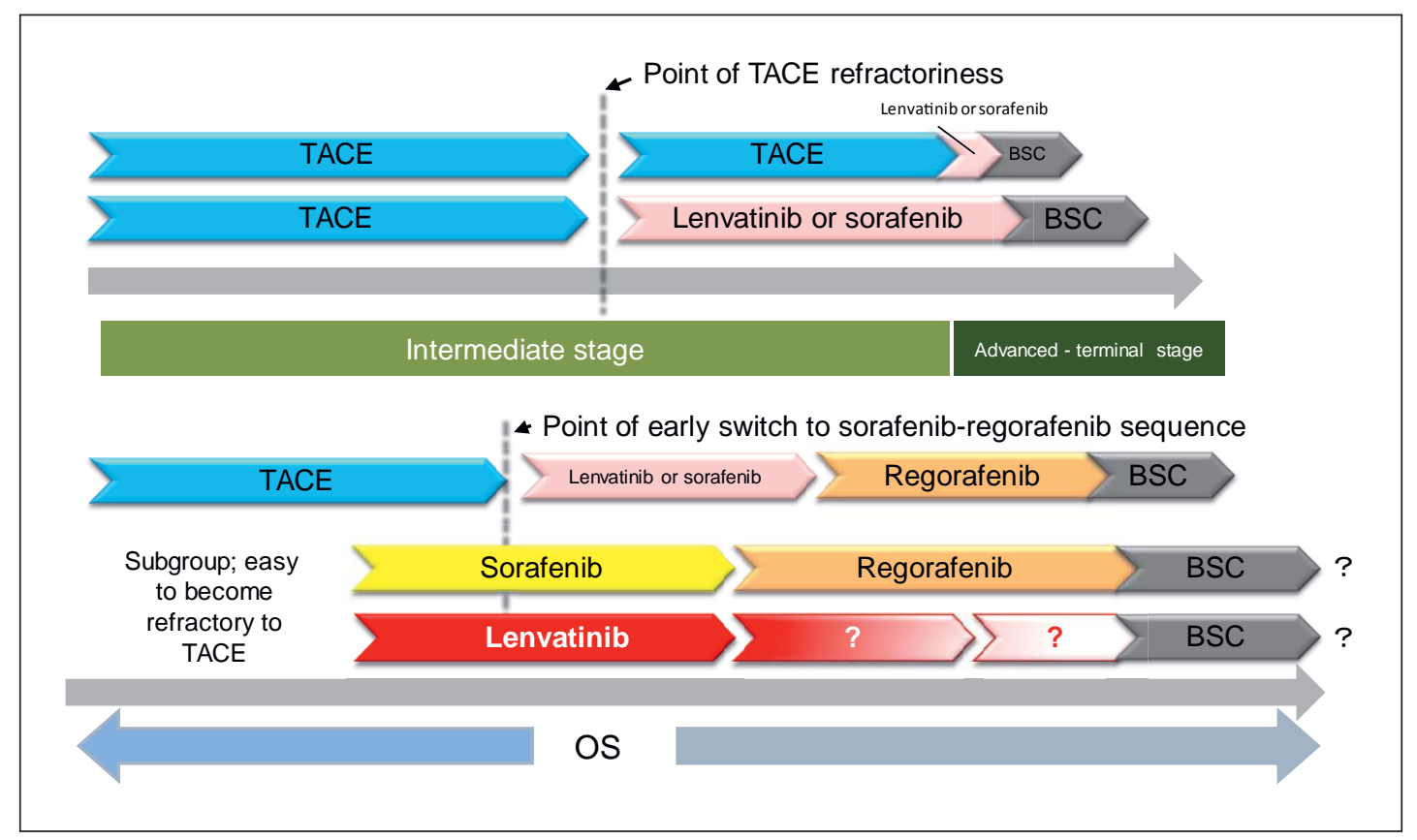

Fig. 1. Treatment strategy for systemic therapy for hepatocellular carcinoma. Identification of the subgroup that easily develops to transarterial chemoembolization (TACE) failure/refractoriness may be important. For that subgroup, systemic therapy with a lenvatinib/sorafenib-regorafenib sequence may be a more adequate treatment strategy than repeating ineffective TACE for improving patient survival/benefit. BSC, best supportive care; OS, overall survival.

\section{A New First-Line Therapy Option: Lenvatinib}

Lenvatinib is an oral multi-tyrosine kinase receptor inhibitor that targets VEGF receptors 1-3, FGFR 1-4, PDGFR $\alpha$, and the KIT and RET proto-oncogenes. It is approved worldwide for the treatment of radioactive iodine-refractory differentiated thyroid cancer. A phase II study evaluating lenvatinib for HCC treatment showed good outcomes, with an mOS of 18.7 months, a time to progression (TTP) of 7.4 months, an objective response rate (ORR) of $37 \%$, and a disease control rate of 78\% (according to the modified Response Evaluation Criteria in Solid Tumors [RECIST]) [22]. Considering that the ORR in the control arm of the BRISK-TA study was $42 \%$, the ORR of $37 \%$ among those treated with lenvatinib may indicate that the levels of tumor necrosis induced by lenvatinib are similar to those induced by TACE (Table 4).

A worldwide press release by Eisai Co., Ltd. (January 25,2017) revealed the results of the phase III REFLECT trial involving 954 patients with unresectable HCC [11]. Achievement of the primary endpoint $(\mathrm{OS})$ in the lenvatinib trial was not inferior to that with sorafenib. The secondary endpoints (progression-free survival, TTP, and ORR) also showed that lenvatinib is statistically superior to sorafenib, with clinical significance. Details will be reported at relevant conferences and in journals. The approval of lenvatinib as a novel agent for unresectable HCC in Asia, Japan, Europe, and the USA is anticipated. The improvement of OS with lenvatinib, which is similar to that reported for sorafenib, will likely lead to the approval of lenvatinib as a first-line agent, in addition to sorafenib, for unresectable HCC.

Once lenvatinib will be approved and will become available for treating HCC patients, criteria for the differential use of lenvatinib and sorafenib in the clinical setting will need to be established. The benefits of sorafenib-regorafenib sequential therapy were demonstrated 
Kudo et al.: A New Era of Systemic Therapy for Hepatocellular Carcinoma with Regorafenib and Lenvatinib

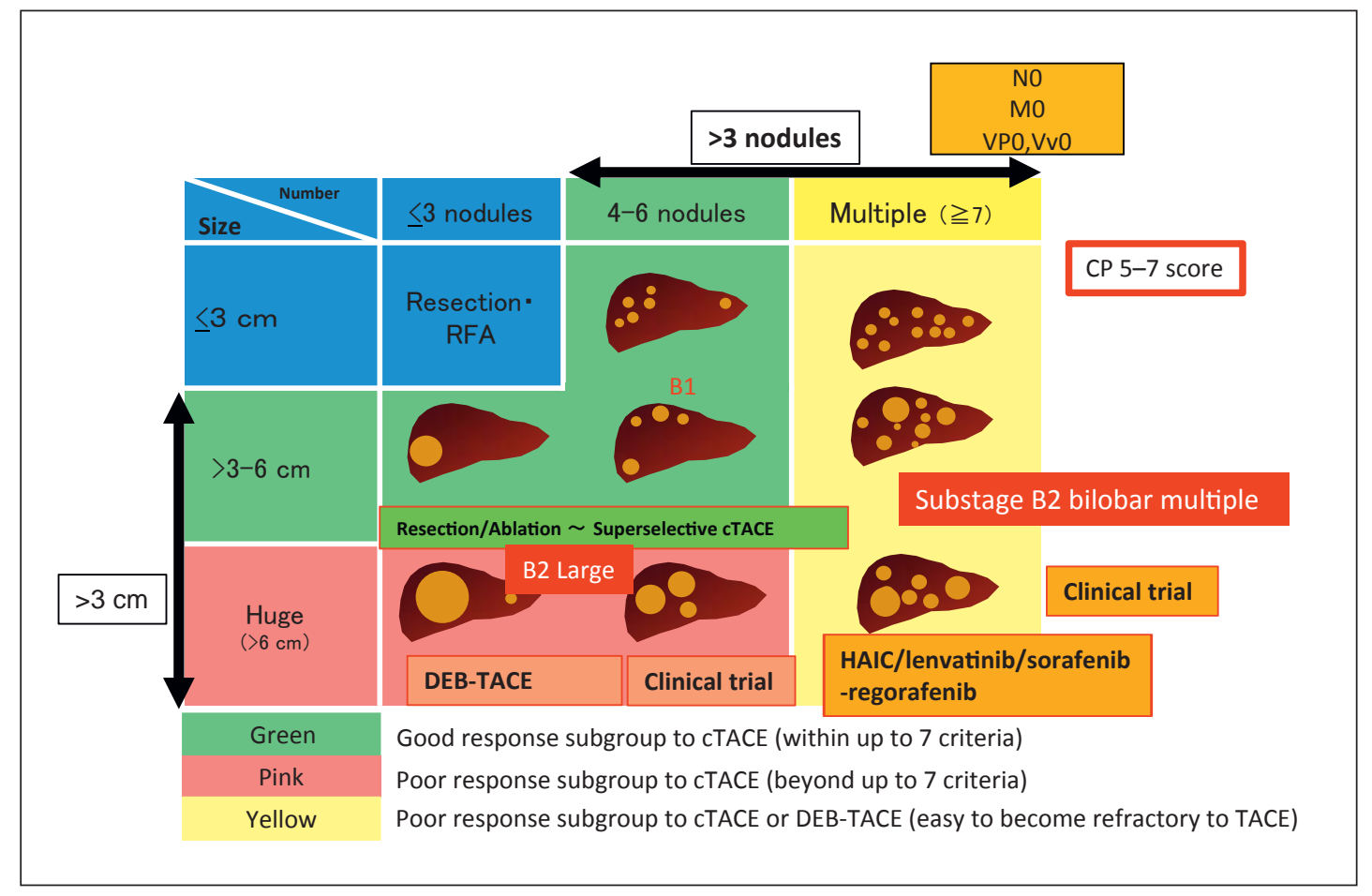

Fig. 2. Heterogeneity and treatment strategy for intermediate-stage hepatocellular carcinoma. Substage B2 (bilobar multiple nodules) may be a candidate for clinical trials of transarterial chemoembolization (TACE) combination therapy with tyrosine kinase inhibitors or immunotherapy. This subgroup may easily become refractory to TACE. CP, Child-Pugh; RFA, radiofrequency ablation; cTACE, conventional subsegmental Lipiodol TACE; DEB, drug-eluting beads; HAIC, hepatic arterial infusion chemotherapy. Modified with permission by Kudo et al. [12].

Table 4. Comparison of ORR and DCR between TACE and lenvatinib

\begin{tabular}{lcc}
\hline & $\begin{array}{l}\text { TACE (placebo arm of } \\
\text { brivanib-TACE trial) [20] }\end{array}$ & $\begin{array}{l}\text { Lenvatinib } \\
\text { (phase II trial) [22] }\end{array}$ \\
\hline $\begin{array}{l}\text { Subjects, } n \\
\text { Child-Pugh class, } n(\%)\end{array}$ & 253 & 46 \\
A & $231(91)$ & $45(97.8)$ \\
B & $20(8)$ & $1(2.2)$ \\
C & $2(1)$ & \\
BCLC stage, $n(\%)$ & $57(23)$ & $0(0)$ \\
A & $150(59)$ & $19(41.3)$ \\
B & $44(17)$ & $27(58.7)$ \\
C & $2(1)$ & $0(0)$ \\
D & $106(42)$ & $17(37)$ \\
ORR, $n(\%)$ & $199(79)$ & $36(78)$ \\
DCR, $n(\%)$ & & \\
\hline
\end{tabular}

ORR, objective response rate; DCR, disease control rate; TACE, transarterial chemoembolization; BCLC, Barcelona Clinic Liver Cancer 
Kudo et al.: A New Era of Systemic Therapy for Hepatocellular Carcinoma with Regorafenib and Lenvatinib

in a solid reliable prospective study $[10,19]$. Second-line therapy with regorafenib resulted in significant improvement in patients who had suffered disease progression while on firstline sorafenib, with an mOS of 26 months from the start of sorafenib therapy, even in a group with predominantly advanced-stage HCC (BCLC-C) [19]. However, it is pertinent to note that the subject population was carefully selected.

On the other hand, lenvatinib showed clinically significant antitumor effects (progressionfree survival, TTP, and ORR) superior to sorafenib, as disclosed by a press release [11] and phase II trial results [22]. This therapeutic agent is expected to be as effective as TACE in terms of reducing tumor size and inducing tumor necrosis. In some cases, it is expected to permit conversion from systemic therapy to TACE or curative options such as resection or ablation [23-26]. This is a potential advantage of lenvatinib.

For all intents and purposes, the arrival of regorafenib and lenvatinib provides the possibility of systemic therapy as an option for a subgroup of patients with intermediate-stage HCC who easily become or are refractory to TACE, in addition to those with advanced-stage HCC. Patients with intermediate-stage HCC who are likely to become refractory to TACE need to be identified to allow proactive intervention with systemic therapy (Fig. 1).

\section{Conclusion}

The results of phase III studies investigating 2 new therapeutic agents for HCC - namely, regorafenib and lenvatinib - were recently disclosed. The former was presented at a conference in June 2016, and results were published in a journal in January 2017; the latter was announced in a press release in January 2017. Sorafenib-regorafenib sequential therapy is of particular interest. The performance of lenvatinib will remain unclear until relevant congresses or publications; however, based on the contents of the press release, it is very likely to be indicated for the first-line treatment of unresectable HCCs. This suggests that additional therapeutic options will become available for patients with intermediate-stage (BCLC-B) as well as advanced-stage (BCLC-C) HCC.

\section{References}

1 Llovet JM, Ricci S, Mazzaferro V, et al: Sorafenib in advanced hepatocellular carcinoma. N Engl J Med 2008;359: 378-390.

2 Cheng AL, Kang YK, Chen Z, et al: Efficacy and safety of sorafenib in patients in the Asia-Pacific region with advanced hepatocellular carcinoma: a phase III randomised, double-blind, placebo-controlled trial. Lancet Oncol 2009;10:25-34.

3 Cheng AL, Kang YK, Lin DY, et al: Sunitinib versus sorafenib in advanced hepatocellular cancer: results of a randomized phase III trial. J Clin Oncol 2013;31:4067-4075.

4 Johnson PJ, Qin S, Park JW, et al: Brivanib versus sorafenib as first-line therapy in patients with unresectable, advanced hepatocellular carcinoma: results from the randomized phase III BRISK-FL study. J Clin Oncol 2013; 31:3517-3524.

5 Cainap C, Qin S, Huang WT, etal: Linifanib versus sorafenib in patients with advanced hepatocellular carcinoma: results of a randomized phase III trial. J Clin Oncol 2015;33:172-179.

6 Llovet JM, Decaens T, Raoul JL, et al: Brivanib in patients with advanced hepatocellular carcinoma who were intolerant to sorafenib or for whom sorafenib failed: results from the randomized phase III BRISK-PS study. J Clin Oncol 2013;31:3509-3516.

7 Zhu AX, Kudo M, Assenat E, et al: Effect of everolimus on survival in advanced hepatocellular carcinoma after failure of sorafenib: the EVOLVE-1 randomized clinical trial. JAMA 2014;312:57-67.

8 Zhu AX, Park JO, Ryoo BY, et al: Ramucirumab versus placebo as second-line treatment in patients with advanced hepatocellular carcinoma following first-line therapy with sorafenib (REACH): a randomised, double-blind, multicentre, phase 3 trial. Lancet Oncol 2015;16:859-870. 
Kudo et al.: A New Era of Systemic Therapy for Hepatocellular Carcinoma with Regorafenib and Lenvatinib

9 Bruix J, Merle P, Granito A, et al: Efficacy and safety of regorafenib versus placebo in patients with hepatocellular carcinoma (HCC) progressing on sorafenib: results of the international, randomized phase 3 RESORCE trial (abstract LBA-03). Ann Oncol 2016;27(suppl 2):ii140-ii141.

10 Bruix J, Qin S, Merle P, et al: Regorafenib for patients with hepatocellular carcinoma who progressed on sorafenib treatment (RESORCE): a randomised, double-blind, placebo-controlled, phase 3 trial. Lancet 2017; 389:56-66.

11 Eisai Co., Ltd.: Phase III trial of anticancer agent Lenvima ${ }^{\circledR}$ as first-line treatment for unresectable hepatocellular carcinoma meets primary endpoint. News Release No. 17-06, January 25, 2017.

12 Kudo M, Arizumi T, Ueshima K, et al: Subclassification of BCLC B stage hepatocellular carcinoma and treatment strategies: proposal of modified Bolondi's subclassification (Kinki criteria). Dig Dis 2015;33:751-758.

13 Arizumi T, Ueshima K, Iwanishi M, et al: Validation of Kinki criteria, a modified substaging system, in patients with intermediate stage hepatocellular carcinoma. Dig Dis 2016;34:671-678.

14 Ogasawara S, Chiba T, Ooka Y, et al: Efficacy of sorafenib in intermediate-stage hepatocellular carcinoma patients refractory to transarterial chemoembolization. Oncology 2014;87:330-341.

15 Arizumi T, Ueshima K, Chishina H, et al: Validation of the criteria of transcatheter arterial chemoembolization failure or refractoriness in patients with advanced hepatocellular carcinoma proposed by the LCSGJ. Oncology 2014;87(suppl 1):32-36.

16 Kudo M, Matsui O, Izumi N, et al: Transarterial chemoembolization failure/refractoriness: JSH-LCSGJ criteria 2014 update. Oncology 2014;87(suppl 1):22-31.

17 Arizumi T, Ueshima K, Minami T, et al: Effectiveness of sorafenib in patients with transcatheter arterial chemoembolization (TACE) refractory and intermediate-stage hepatocellular carcinoma. Liver Cancer 2015;4:253262.

18 Kudo M: Regorafenib as second-line systemic therapy may change the treatment strategy and management paradigm for hepatocellular carcinoma. Liver Cancer 2016;5:235-244.

19 Finn RS, Merle P, Granito A, et al: Outcomes with sorafenib (SOR) followed by regorafenib (REG) or placebo (PBO) for hepatocellular carcinoma (HCC): results of the international, randomized phase 3 RESORCE trial. J Clin Oncol 2017;35(suppl 4S); abstract 344.

20 Kudo M, Han G, Finn RS, et al: Brivanib as adjuvant therapy to transarterial chemoembolization in patients with hepatocellular carcinoma: a randomized phase III trial. Hepatology 2014;60:1697-1707.

21 Kudo M, Imanaka K, Chida N, et al: Phase III study of sorafenib after transarterial chemoembolisation in Japanese and Korean patients with unresectable hepatocellular carcinoma. Eur J Cancer 2011;47:2117-2127.

22 Ikeda K, Kudo M, Kawazoe S, et al: Phase 2 study of lenvatinib in patients with advanced hepatocellular carcinoma. J Gastroenterol 2016, Epub ahead of print.

23 Kudo M, Izumi N, Sakamoto M, et al: Survival analysis over 28 years of 173,378 patients with hepatocellular carcinoma in Japan. Liver Cancer 2016;5:190-197.

24 Kudo M. Surveillance, diagnosis, treatment, and outcome of liver cancer in Japan. Liver Cancer 2015;4:39-50.

25 Kudo M: Locoregional therapy for hepatocellular carcinoma. Liver Cancer 2015;4:163-164.

26 Tsurusaki M, Murakami T: Surgical and locoregional therapy of HCC: TACE. Liver Cancer 2015;4:165-175. 